\title{
Searches for New Quarks and Leptons Produced in $Z$-Boson Decay
}

G. S. Abrams, ${ }^{(1)}$ C. E. Adolphsen, ${ }^{(2)}$ D. Averill, ${ }^{(3)}$ J. Ballam, ${ }^{(4)}$ B. C. Barish, ${ }^{(5)}$ T. Barklow, ${ }^{(4)}$ B. A. Barnett, ${ }^{(6)}$ J. Bartelt, ${ }^{(4)}$ S. Bethke, ${ }^{(1)}$ D. Blockus, ${ }^{(3)}$ G. Bonvicini, ${ }^{(7)}$ A. Boyarski, ${ }^{(4)}$ B. Brabson, ${ }^{(3)}$ A. Breakstone, ${ }^{(8)}$ F. Bulos, ${ }^{(4)}$ P. R. Burchat, ${ }^{(2)}$ D. L. Burke, ${ }^{(4)}$ R. J. Cence, ${ }^{(8)}$ J. Chapman, ${ }^{(7)}$ M. Chmeissani, ${ }^{(7)}$ D. Cords, ${ }^{(4)}$ D. P. Coupal, ${ }^{(4)}$ P. Dauncey, ${ }^{(6)}$ H. C. DeStaebler, ${ }^{(4)}$ D. E. Dorfan, ${ }^{(2)}$ J. M. Dorfan, ${ }^{(4)}$ D. C. Drewer, ${ }^{(6)}$ R. Elia, ${ }^{(4)}$ G. J. Feldman, ${ }^{(4)}$ D. Fernandes, ${ }^{(4)}$ R. C. Field, ${ }^{(4)}$ W. T. Ford, ${ }^{(9)}$ C. Fordham, ${ }^{(4)}$ R. Frey, ${ }^{(7)}$ D. Fujino, ${ }^{(4)}$ K. K. Gan, ${ }^{(4)}$ E. Gero, ${ }^{(7)}$ G. Gidal, ${ }^{(1)}$ T. Glanzman, ${ }^{(4)}$ G. Goldhaber, (1) J. J. Gomez Cadenas, ${ }^{(2)}$ G. Gratta, ${ }^{(2)}$ G. Grindhammer, ${ }^{(4)}$ P. Grosse-Wiesmann, ${ }^{(4)}$ G. Hanson, ${ }^{(4)}$ R. Harr, ${ }^{(1)}$ B. Harral, ${ }^{(6)}$ F. A. Harris, ${ }^{(8)}$ C. M. Hawkes, ${ }^{(5)}$ K. Hayes, ${ }^{(4)}$ C. Hearty, ${ }^{(1)}$ C. A. Heusch, ${ }^{(2)}$ M. D. Hildreth, ${ }^{(4)}$ T. Himel, ${ }^{(4)}$ D. A. Hinshaw, ${ }^{(9)}$ S. J. Hong, ${ }^{(7)}$ D. Hutchinson, ${ }^{(4)}$ J. Hylen, ${ }^{(6)}$ W. R. Innes, ${ }^{(4)}$ R. G. Jacobsen, ${ }^{(4)}$ J. A. Jaros, ${ }^{(4)}$ C. K. Jung, ${ }^{(4)}$ J. A. Kadyk, ${ }^{(1)}$ J. Kent, ${ }^{(2)}$ M. King, ${ }^{(2)}$ S. R. Klein, ${ }^{(4)}$ D. S. Koetke, ${ }^{(4)}$ S. Komamiya, ${ }^{(4)}$ W. Koska, ${ }^{(7)}$ L. A. Kowalski, ${ }^{(4)}$ W. Kozanecki, ${ }^{(4)}$ J. F. Kral, ${ }^{(1)}$ M. Kuhlen, ${ }^{(5)}$ L. Labarga, ${ }^{(2)}$ A. J. Lankford, ${ }^{(4)}$ R. R. Larsen, ${ }^{(4)}$ F. Le Diberder, ${ }^{(4)}$ M. E. Levi, (1) A. M. Litke, ${ }^{(2)}$ X. C. Lou, ${ }^{(3)}$ V. Lüth, ${ }^{(4)}$ J. A. McKenna, ${ }^{(5)}$ J. A. J. Matthews, ${ }^{(6)}$ T. Mattison, ${ }^{(4)}$ B. D. Milliken, ${ }^{(5)}$ K. C. Moffeit, ${ }^{(4)}$ C. T. Munger, ${ }^{(4)}$ W. N. Murray, ${ }^{(3)}$ J. Nash, ${ }^{(4)}$ H. Ogren, ${ }^{(3)}$ K. F. O'Shaughnessy, ${ }^{(4)}$ S. I. Parker, ${ }^{(8)}$ C. Peck, ${ }^{(5)}$ M. L. Perl, ${ }^{(4)}$ F. Perrier, ${ }^{(4)}$ M. Petradza, ${ }^{(7)}$ R. Pitthan, ${ }^{(4)}$ F. C. Porter, ${ }^{(5)}$ P. Rankin, ${ }^{(9)}$ K. Riles, ${ }^{(4)}$ F. R. Rouse, ${ }^{(4)}$ D. R. Rust, ${ }^{(3)}$ H. F. W. Sadrozinski, ${ }^{(2)}$ M. W. Schaad, (1) B. A. Schumm, ${ }^{(1)}$ A. Seiden, ${ }^{(2)}$ J. G. Smith, ${ }^{(9)}$ A. Snyder, ${ }^{(3)}$ E. Soderstrom, ${ }^{(5)}$ D. P. Stoker, ${ }^{(6)}$ R. Stroynowski, ${ }^{(5)}$ M. Swartz, ${ }^{(4)}$ R. Thun, ${ }^{(7)}$ G. H. Trilling, ${ }^{(1)}$ R. Van Kooten, ${ }^{(4)}$ P. Voruganti, ${ }^{(4)}$ S. R. Wagner, ${ }^{(9)}$ S. Watson, ${ }^{(2)}$ P. Weber, ${ }^{(9)}$ A. Weigend, ${ }^{(4)}$ A. J. Weinstein, ${ }^{(5)}$ A. J. Weir, ${ }^{(5)}$ E. Wicklund, ${ }^{(5)}$ M. Woods, ${ }^{(4)}$ D. Y. Wu, ${ }^{(5)}$ M. Yurko, ${ }^{(3)}$ C. Zaccardelli, ${ }^{(2)}$ and C. von Zanthier ${ }^{(2)}$

${ }^{(1)}$ Lawrence Berkeley Laboratory and Department of Physics, University of California, Berkeley, California 94720

${ }^{(2)}$ University of California, Santa Cruz, California 95064

${ }^{(3)}$ Indiana University, Bloomington, Indiana 47405

${ }^{(4)}$ Stanford Linear Accelerator Center, Stanford University, Stanford, California 94309

${ }^{(5)}$ California Institute of Technology, Pasadena, California 91125

${ }^{(6)}$ Johns Hopkins University, Baltimore, Maryland 21218

(7) University of Michigan, Ann Arbor, Michigan 48109

${ }^{(8)}$ University of Hawaii, Honolulu, Hawaii 96822

${ }^{(9)}$ University of Colorado, Boulder, Colorado 80309 (Received 13 October 1989)

\begin{abstract}
We have searched for events with new-particle topologies in 390 hadronic $Z$ decays with the Mark II detector at the SLAC Linear Collider. We place 95\%-confidence-level lower limits of $40.7 \mathrm{GeV} / \mathrm{c}^{2}$ for the top-quark mass, $42.0 \mathrm{GeV} / \mathrm{c}^{2}$ for the mass of a fourth-generation charge $-\frac{1}{3}$ quark, and 41.3 $\mathrm{GeV} / \mathrm{c}^{2}$ for the mass of an unstable Dirac neutral lepton.
\end{abstract}

PACS numbers: $13.38 .+\mathrm{c}, 12.15 . \mathrm{Ff}, 14.60 . \mathrm{Gh}, 14.80 . \mathrm{Dq}$

We have searched for new quarks and leptons in $Z$ boson decay using data from the Mark II detector at the SLAC $e^{+} e^{-}$Linear Collider (SLC) operating in the $e^{+} e^{-}$center-of-mass energy $\left(E_{\text {c.m. }}\right)$ range from 89.2 to 93.0 GeV. The standard model predicts the existence of the top quark and does not exclude the possibility of new generations of fermions. The existence and masses of these possible new particles may provide information to help understand the pattern of fermion masses and the presence of generations.

We specifically search for top $(t)$ quarks, fourthgeneration charge $-\frac{1}{3}\left(b^{\prime}\right)$ quarks, and unstable neutral Dirac leptons $\left(L^{0}\right)$; the small production rate of heavy sequential charged leptons on the $Z$-boson resonance prevents us from searching for sequential charged leptons at this time. We assume pair production of the new particles through $Z$ decay with couplings and decay widths given by the standard model.

One expects $t$ quarks to decay via the virtual- $W\left(W^{*}\right)$ charged-current (CC) process $t \rightarrow b W^{*}$. Naively, one also expects the $b^{\prime}$ quark to decay via the CC process $b^{\prime} \rightarrow c W^{*}$ if the mass of the $b^{\prime}$ quark is less than the mass of the $t$ quark $\left(M_{b^{\prime}}<M_{t}\right)$.. Assuming that a new quark decays $100 \%$ via the CC mode, the UA1 Collaboration ${ }^{1}$ has excluded top quarks with masses less than $44 \mathrm{GeV} / c^{2}$ and $b^{\prime}$ quarks with masses less than 32 $\mathrm{GeV} / \mathrm{c}^{2}$. With the same assumption, the CDF Collaboration ${ }^{2}$ has excluded top quarks with masses between 40 and $77 \mathrm{GeV} / c^{2}$.

A $b^{\prime}$ quark may not decay $100 \%$ of the time via the CC decay because of increased suppression of transitions which cross two generations. ${ }^{3}$ Consequently, the flavorchanging neutral-current (FCNC) loop decays ${ }^{4}$ of $b^{\prime} \rightarrow b+$ gluon and $b^{\prime} \rightarrow b \gamma$ must also be considered. 
Furthermore, in extensions of the standard model with two Higgs doublets, $t$ and $b^{\prime}$ can decay into charged Higgs particles $\left(H^{+}\right)$by $t \rightarrow H^{+} b$ or $b^{\prime} \rightarrow H^{-} c$ if $M_{H^{ \pm}}<M_{t}, M_{b^{\prime}}$. This two-body decay mode would dominate over the CC decay. Independent of decay mode, a $b^{\prime}$ quark has been excluded for masses less than 25.6 $\mathrm{GeV} / c^{2}$ by the VENUS Collaboration ${ }^{5}$ at the KEK $e^{+} e^{-}$storage ring TRISTAN.

We restrict our $L^{0}$ search to a sequential fourthgeneration Dirac neutral lepton. We assume that $M_{L^{0}}$ $<M_{L^{-}}$in the new lepton doublet $\left(L^{0}, L^{-}\right)$, and that the weak eigenstates $v_{l}$ and mass eigenstates $L_{i}^{0}$ of the four generations of neutrinos are mixed in analogy with the quark sector:

$$
v_{l}=\sum_{i=1}^{4} U_{l i} L_{i}^{0}
$$

The possible decay modes of the $L^{0}$ are then $L^{0} \rightarrow l$ $+W^{*}(l=e, \mu, \tau)$. Dirac neutral leptons which decay via $L^{0} \rightarrow e^{-}+W^{*}$ or $L^{0} \rightarrow \mu^{-}+W^{*}$ have been excluded for masses less than $27 \mathrm{GeV} / \mathrm{c}^{2}$ by the AMY Collaboration. $^{6}$

We have investigated three types of event topologies. Type 1 is an event with an isolated charged track. The semileptonic decays of $t$ and $b^{\prime}$ or the decays of $L^{0}$ will produce isolated leptons. To keep detection efficiencies high, lepton identification is not used. The type- 2 topology is an event with an isolated photon. The decay $b^{\prime} \rightarrow b \gamma$ motivates the search for this topology. Type 3 is the topology produced by a pair of heavy objects each decaying hadronically into two or more jets. Massive particles decaying into jets tend to produce spherical events which can be characterized by large momentum sums out of the event plane. The decay $b^{\prime} \rightarrow b+$ gluon, $t$ decaying through $\mathrm{H}^{+} b$, with the $\mathrm{H}^{+}$decaying $100 \%$ hadronically, and the $\mathrm{CC}$ hadronic decays of $t$ and $b^{\prime}$ are examples.

Details of the Mark II detector can be found elsewhere. ${ }^{7}$ A cylindrical drift chamber in a $4.75-\mathrm{kG}$ axial magnetic field measures charged-particle momenta. Photons are detected in electromagnetic calorimeters covering the angular region $|\cos \theta|<0.96$, where $\theta$ is the angle with respect to the beam axis. Barrel leadliquid-argon sampling calorimeters cover the central region $|\cos \theta|<0.72$ and the remaining solid angle is covered by end-cap lead-proportional-tube calorimeters. The detector is triggered by two or more charged tracks within $|\cos \theta|<0.76$ or by neutral-energy requirements of a single shower depositing at least $3.3 \mathrm{GeV}$ in the barrel calorimeter or $2.2 \mathrm{GeV}$ in an end-cap calorimeter. This combination results in an estimated trigger efficiency of greater than $99 \%$ for hadronic $Z$ decays.

All three types of event topologies share the following event-selection criteria: Charged tracks are required to project into a cylindrical volume of radius $1 \mathrm{~cm}$ and halflength of $3 \mathrm{~cm}$ around the nominal collision point parallel to the beam axis, to be within the angular region $|\cos \theta|<0.85$, and to have to transverse momenta with respect to the beam axis of at least $150 \mathrm{MeV} / c$. An electromagnetic shower is required to have shower energy greater than $1 \mathrm{GeV}$ and $|\cos \theta|<0.68$ for the central calorimeter and $0.68<|\cos \theta|<0.95$ for the end-cap calorimeter. All events are required to contain at least six charged tracks and the sum of charged-particle energy and shower energy $\left(E_{\text {vis }}\right)$ must be greater than $0.1 E_{\text {c.m. }}$. To ensure that the events are well contained within the detector, the polar angle of the thrust axis $\left(\theta_{\mathrm{thr}}\right)$ of each event must satisfy the condition $\left|\cos \theta_{\mathrm{thr}}\right|$ $<0.8$.

The expected number of produced hadronic events before cuts is normalized to the total number of hadronic events $\left(N_{h}\right)$ that fulfill the hadronic-event-selection criteria described in a previous Letter. ${ }^{8}$ The expected number of produced exotic events $N_{x}, x=t, b^{\prime}$, or $L^{0}$, is given by

$$
N_{x}=N_{h} \Gamma_{x} /\left(\epsilon_{q} \Gamma_{q}+\epsilon_{x} \Gamma_{x}\right),
$$

where $\Gamma_{q}$ is the partial width of the $Z$ to $u, d, s, c$, and $b$ (udscb) quarks, $\epsilon_{q}=0.953$ is the efficiency for $u d s c b$ quarks to pass the hadronic event criteria, $\Gamma_{x}$ is the partial width of the $Z$ to the exotic particle in question, and $\epsilon_{x}$ is the efficiency for the exotic particle events to pass the hadronic event criteria. First-order QCD corrections ${ }^{9}$ are used when calculating $\Gamma_{q}$ and $\Gamma_{x}$. The data sample consists of $N_{h}=390$ events, corresponding to an integrated luminosity of $16.7 \pm 0.8 \mathrm{nb}^{-1}$.

Type- 1 events must have event thrust less than 0.9 and must contain at least one isolated charged track. An isolated track is one with isolation parameter $\rho_{i}>1.8$, where $\rho_{i}$ is defined as follows: the LUND jet-finding algorithm is applied ${ }^{10}$ to the charged and neutral tracks excluding the candidate track $i$. We then define

$$
\rho_{i} \equiv \min _{\text {jets } j}\left\{\left[2 E_{i}\left(1-\cos \theta_{i j}\right)\right]^{1 / 2}\right\} \text {, }
$$

where $E_{i}$ is the track energy in $\mathrm{GeV}$ and $\theta_{i j}$ is the angle

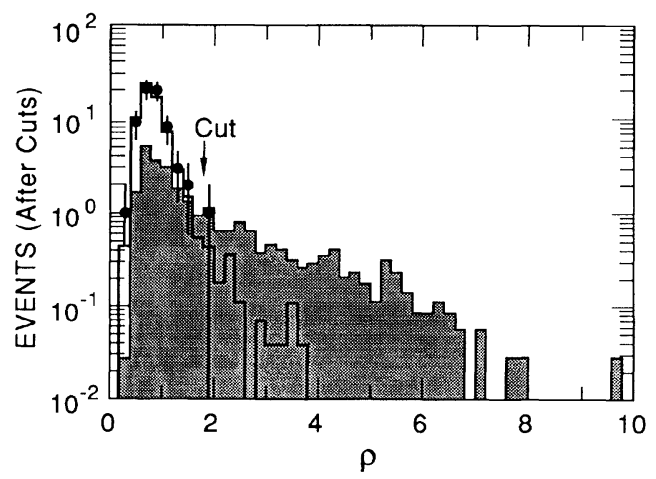

FIG. 1. Maximum isolation parameter $\rho$ of all the tracks in an event for data (circles, with statistical errors), $u d s c b$ QCD Monte Carlo simulation (solid line), and a $35-\mathrm{GeV} / \mathrm{c}^{2}$ top quark (shaded area, normalized to data). The Monte Carlo simulation includes detector and beam background effects. 


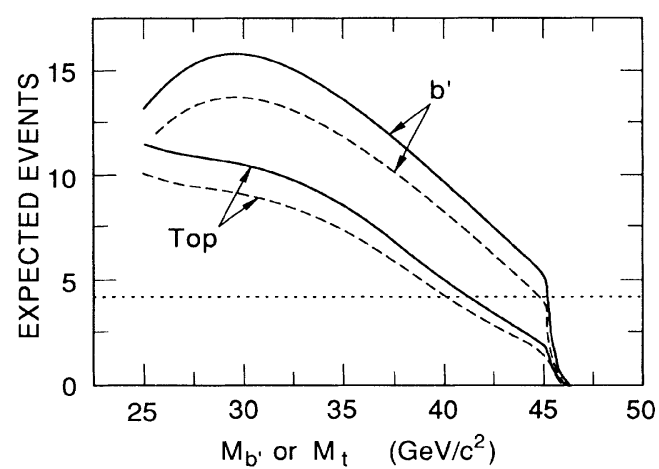

FIG. 2. Expected number of top and $b^{\prime}$ (decaying $100 \%$ via CC process) quark events with at least one isolated $\left(\rho_{i}>1.8\right)$ track as a function of the quark mass (solid curves). The dashed curves indicate the central value minus the uncertainty from statistical and systematic errors. The dotted line is the upper bound at $95 \%$ C.L. with background subtracted for the observed number of events.

between the track and each jet axis. The distribution of $\rho$, the maximum value of $\rho_{i}$ of all charged tracks in an event, is shown in Fig. 1 for our data sample, for a fiveflavor QCD Monte Carlo simulation, ${ }^{11}$ and for a 35$\mathrm{GeV} / c^{2} t$ quark (CC decay).

The detection efficiencies $\left(\epsilon_{D}\right)$ for $t, b^{\prime}$, and $L^{0}$ are calculated with the LUND 6.3 parton-shower Monte Carlo program with LUND symmetric fragmentation. ${ }^{11}$ Uncertainties in detection efficiency $\left(\Delta \epsilon_{D} / \epsilon_{D}\right)$ from Monte Carlo statistics $(\approx 3 \%)$, detector simulation and beam backgrounds $(\approx 1 \%)$, theoretical uncertainties ${ }^{12}$ in semileptonic branching ratios $(\approx 2 \%)$, and fragmentation models are calculated. The last error is estimated using different Monte Carlo generators and fragmentation schemes, ${ }^{13}$ and is found to vary strongly with heavyquark mass. For masses approaching the beam energy the error is negligible, while for masses in the range $25-30 \mathrm{GeV} / \mathrm{c}^{2}$ the error can be as large as $12 \%$. We choose to use the value $12 \%$ for all quark masses.

The number of produced events $N_{x}$ has both a statistical uncertainty from $N_{h}$, and a substantial systematic error (as large as $25 \%$ depending on the exotic particle mass) due to uncertainties in higher-order QCD corrections $^{9}$ in the calculation of $\Gamma_{x}$ if $x$ is a $t$ or $b^{\prime}$ quark.

The total error on the expected number of events is calculated by summing the individual statistical and maximum systematic errors in quadrature. The lower solid curve in Fig. 2 shows our best estimate of the expected number of top events with at least one isolated track as a function of the top-quark mass. The dashed curve is our best estimate minus the total error and is used for setting mass limits. The corresponding curves for $b^{\prime} \rightarrow c W^{*}$ are also shown.

There is one event satisfying the type- 1 event-selection criteria in our data sample of 390 hadronic $Z$ decays, while 0.9 event (LUND shower with Peterson fragmentation ${ }^{13}$ ) to 1.8 events (WEBBER $4.1^{13}$ ) are expected from

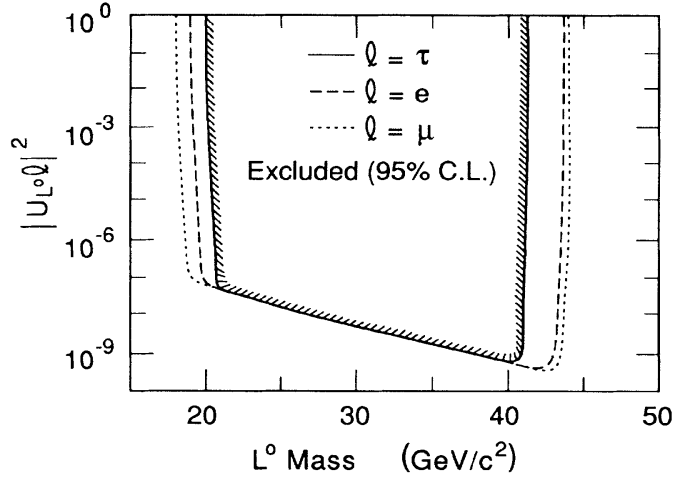

FIG. 3. 95\%-C.L. mass limits for an unstable neutral heavy lepton $L^{0}$ as a function of mass and mixing-matrix element $\left|U_{L^{0} l}\right|^{2}$ for $B\left(L^{0} \rightarrow \tau W^{*}\right)=100 \%, B\left(L^{0} \rightarrow e W^{*}\right)=100 \%$, and $B\left(L^{0} \rightarrow \mu W^{*}\right)=100 \%$, as indicated.

QCD five-flavor processes. To be conservative, background subtraction is performed using the smallest value (0.9 event) expected. Using a standard approach, ${ }^{14}$ we find the upper limit at $95 \%$ C.L. to be 4.2 events for one observed event and 0.9 expected background event.

From the above observation, we conclude that $\boldsymbol{M}_{t}$ $>40.0 \mathrm{GeV} / c^{2}$ and $M_{b^{\prime}}>44.7 \mathrm{GeV} / c^{2}$ at the $95 \%$ confidence level if $t$ and $b^{\prime}$ decay $100 \%$ via the CC process.

Limits on $L^{0}$ production are sensitive to the mixing parameter $U_{L} \%$. For small enough values of the sum $\sum\left|U_{L}{ }^{0}\right|^{2}$ for $l=e, \mu, \tau$, the lifetime ${ }^{15}$ of the $L^{0}$ will be sufficiently long that it will decay outside our fiducial vertex region. Such decays may produce spectacular signatures, but they are not the subject of this Letter. We therefore obtain the $L^{0}$ mass limits shown in Fig. 3 .

For the type-2 event topology we require that the event thrust not exceed 0.9 and that there be at least one isolated photon. An isolated photon is defined as a neutral shower with $\rho_{i}>3.0$, where $\rho_{i}$ is defined as for a charged track. A larger $\rho$ cut is required because the calorimeters cannot resolve closely spaced $\pi^{0}$ 's as well as the drift chamber can resolve closely spaced charged pions.

No events were found satisfying the type-2 eventselection criteria. From this observation, we obtain $M_{b^{\prime}}>45.4 \mathrm{GeV} / c^{2}(95 \%$ C.L. $)$ if $B\left(b^{\prime} \rightarrow b \gamma\right) \geq 25 \%$.

The type-3 even topology requires $M_{\text {out }}>18 \mathrm{GeV} / c^{2}$, where

$$
M_{\text {out }} \equiv \frac{E_{\text {c.m. }}}{E_{\text {vis }}} \frac{1}{c} \sum\left|p_{T}^{\text {out }}\right| .
$$

$p_{T}^{\text {out }}$ is the momentum component of a charged track or neutral shower out of the event plane defined by the sphericity tensor, and the sum is over all charged tracks and neutral showers. The distribution of $M_{\text {out }}$ is shown in Fig. 4 for the data sample, for a five-flavor QCD Monte Carlo simulation, and for the process $b^{\prime} \rightarrow c H^{-}$ $\rightarrow c \bar{c} s$ for a $35-\mathrm{GeV} / c^{2} b^{\prime}$.

Six events are observed in the data with $M_{\text {out }}>18$ 


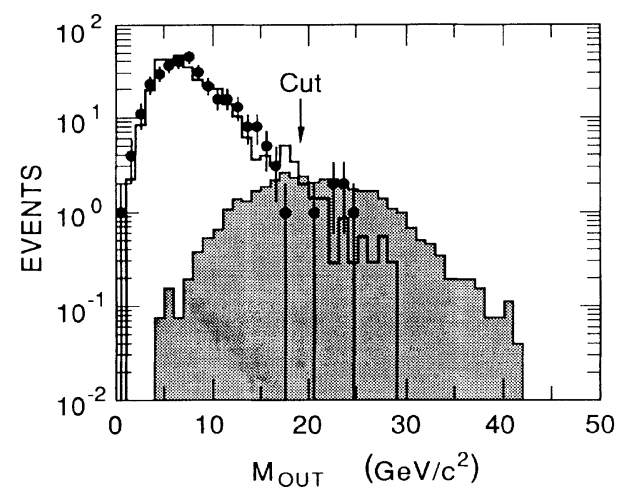

FIG. 4. Mass out of the event plane $M_{\text {out }}$ for data (circles, with statistical errors), udscb QCD Monte Carlo simulation (solid line), and for a $35-\mathrm{GeV} / c^{2} b^{\prime}$ quark decaying into $c H^{-}$ (shaded area), with $M_{H^{-}}=25 \mathrm{GeV} / c^{2}$ and the $H^{-}$decaying $100 \%$ into $\bar{c} s$.

$\mathrm{GeV} / c^{2}$, while 4.8 events (LUND matrix element ${ }^{13}$ ) to 11.7 events (WEBBER $4.1^{13}$ ) are expected from QCD five-flavor processes. The tail of the QCD $M_{\text {out }}$ distribution is very model dependent because of the different ways in which multiple-hard-gluon radiation is handled. ${ }^{13}$ To be conservative, background subtraction is performed using the smallest value (4.8 events) expected. We find the upper limit ${ }^{14}$ at $95 \%$ C.L. to be 7.4 events for 6 observed events and 4.8 expected background events.

The above observation allows us to set the following limits. If $t$ and $b^{\prime}$ decay $100 \%$ via the CC process, $M_{t}>40.7 \mathrm{GeV} / c^{2}$ and $M_{b^{\prime}}>44.2 \mathrm{Gev} / c^{2}$ at $95 \%$ C.L. Note that these limits are more sensitive to the hadronic decays of $t$ and $b^{\prime}$, while the type- 1 event limits are sensitive to the semileptonic decays. If $b^{\prime}$ decays $100 \%$ into $b+$ gluon, then $M_{b^{\prime}}>42.7 \mathrm{GeV} / c^{2}$ at $95 \%$ C.L. If $t$ and $b^{\prime}$ decay $100 \%$ through a charged Higgs boson of mass $\geq 25 \mathrm{GeV} / c^{2}$, which in turn decays $100 \%$ hadronically into $c \bar{s}$, then $M_{t}>42.5 \mathrm{GeV} / c^{2}$ and $M_{b^{\prime}}>45.2 \mathrm{GeV} / c^{2}$ at $95 \%$ C.L. The case of the $H^{-}$decaying partially into $\tau \bar{\nu}$ is found to weaken the above limits, but if $B\left(H^{-} \rightarrow \tau \bar{v}\right)<70 \%$ both limits remain over $40 \mathrm{GeV} / \mathrm{c}^{2}$.

The various mass limits obtained with the three event topologies are summarized in Table I. Finally, the analyses of the three event topologies can be combined to give mass limits on $b^{\prime}$ as a function of branching ratio into the $\mathrm{CC}$ process, assuming that the remaining decays are only through the FCNC decays $b+$ gluon and $b \gamma$ (we assume a Higgs particle is not kinematically accessible). Detection efficiences are found for the possible combinations of the above decays, and combined to give the result that $M_{b^{\prime}}>42.0 \mathrm{GeV} / c^{2}$ (95\% C.L.) for all possible values of the branching ratio into the $\mathrm{CC}$ process and all possible mixtures of $b+$ gluon and $b \gamma$ in the FCNC part.

We express our appreciation to the dedicated efforts of the staff of SLAC and collaborating universities who made the SLC and these results possible. This work was
TABLE I. Summary of the mass limits set by searching for the three event topologies described in the text. For $t \rightarrow b H^{+}$ and $b^{\prime} \rightarrow c H^{-}$limits, $M_{H^{ \pm}} \geq 25 \mathrm{GeV} / c^{2}$. The $L^{0}$ limits are for decays with vertices within the described cylindrical fiducial region (i.e., decay length less than $\approx 1 \mathrm{~cm}$ ).

\begin{tabular}{|c|c|c|c|}
\hline Particle & $\begin{array}{l}\text { Decay products } \\
\quad(B 100 \%)\end{array}$ & Topology & $\begin{array}{c}\text { Mass limit } \\
(95 \% \text { C.L. }) \\
\left(\mathrm{GeV} / c^{2}\right)\end{array}$ \\
\hline \multirow[t]{3}{*}{ Top } & $b W^{*}$ & Isolated track & 40.0 \\
\hline & $b W^{*}$ & $M_{\text {out }}$ & 40.7 \\
\hline & $b H^{+}$ & $M_{\text {out }}$ & 42.5 \\
\hline \multirow[t]{5}{*}{$b^{\prime}$} & $c W^{*}$ & Isolated track & 44.7 \\
\hline & $c W^{*}$ & $M_{\text {out }}$ & 44.2 \\
\hline & $\mathrm{cH}^{-}$ & $M_{\text {out }}$ & 45.2 \\
\hline & $b+$ gluon & $M_{\text {out }}$ & 42.7 \\
\hline & $b \gamma, B \geq 25 \%$ & Isolated photon & 45.4 \\
\hline \multirow[t]{3}{*}{$L^{0}$} & $e W^{*}$ & Isolated track & 43.7 \\
\hline & $\mu W^{*}$ & Isolated track & 44.0 \\
\hline & $\tau W^{*}$ & Isolated track & 41.3 \\
\hline
\end{tabular}

supported in part by Department of Energy Contracts No. DE-AC03-81ER40050 (California Institute of Technology), No. DE-AM03-76SF00010 (University of California, Santa Cruz), No. DE-AC02-86ER40253 (University of Colorado), No. DE-AC03-83ER40103 (University of Hawaii), No. DE-AC02-84ER40125 (Indiana University), No. DE-AC03-76SF00098 (LBL), No. DE-AC02-76ER01112 (University of Michigan), and No. DE-AC03-76SF00515 (SLAC), and by the National Science Foundation (Johns Hopkins University).

${ }^{1}$ C. Albajar et al., Z. Phys. C 37, 505 (1988).

${ }^{2}$ F. Abe et al., University of Pennsylvania Report No. UPR0172E (to be published).

${ }^{3}$ V. Barger et al., Phys. Rev. D 30, 947 (1984).

${ }^{4}$ W. Hou and R. G. Stuart, Phys. Rev. Lett. 62, 617 (1989); V. Barger et al., Phys. Rev. Lett. 57, 1518 (1986).

${ }^{5} \mathrm{~K}$. Abe et al., KEK Report No. 89-39 (to be published). ${ }^{6}$ Y. Sakai et al., KEK Report No. 89-42 (to be published).

${ }^{7}$ G. S. Abrams et al., Nucl. Instrum. Methods Phys. Res., Sect. A 281, 55 (1989).

${ }^{8}$ G. S. Abrams et al., Phys. Rev. Lett. 63, 724 (1989).

${ }^{9}$ J. H. Kühn, A. Reiter, and P. M. Zerwas, Nucl. Phys. B272, 560 (1986).

${ }^{10}$ T. Sjöstrand, Comput. Phys. Commun. 28, 229 (1983). In the algorithm, the jet-forming cutoff parameter $d_{\text {join }}$ is changed from its default value to $d_{\text {join }}=0.5 \mathrm{GeV}$.

${ }^{11}$ LUND 6.3 parton-shower model, T. Sjöstrand, Comput. Phys. Commun. 39, 347 (1986); M. Bengtsson and T. Sjöstrand, Nucl. Phys. B289, 810 (1987). In the hadronic decay of $t$ and $b^{\prime}$ quarks, gluon-emission effects are incorporated by the parton-shower model based on a leading-log approximation. 
${ }^{12}$ M. Jezabek and J. H. Kühn, Phys. Lett. B 207, 91 (1988).

${ }^{13}$ LUND 6.3 parton shower (Ref. 11) and LUND 6.3 matrix element [T. D. Gottschalk and M. P. Shatz, Phys. Lett. 150B, 451

(1985)], both with LUND symmetric fragmentation (Ref. 11) and Peterson fragmentation [C. Peterson et al., Phys. Rev. D 27, 105 (1983)]; and WEBBER 4.1 [G. Marchesini and B. R.
Webber, Nucl. Phys. B238, 1 (1984); B. R. Webber, Nucl. Phys. B238, 492 (1984)].

${ }^{14}$ Particle Data Group, G. P. Yost et al., Phys. Lett. B 204, 81 (1988); O. Helene, Nucl. Instrum. Methods 212, 319 (1983).

${ }^{15}$ F. Gilman, Comments Nucl. Part. Phys. 16, 231 (1986). 